PROCEEDINGS OF THE AMERICAN MATHEMATICAL SOCIETY

Volume 135, Number 4, April 2007, Pages 1123-1127

S 0002-9939(06)08550-9

Article electronically published on October 4, 2006

\title{
BOUND STATES OF DISCRETE SCHRÖDINGER OPERATORS WITH SUPER-CRITICAL INVERSE SQUARE POTENTIALS
}

\author{
DAVID DAMANIK AND GERALD TESCHL
}

(Communicated by Joseph A. Ball)

\begin{abstract}
We consider discrete one-dimensional Schrödinger operators whose potentials decay asymptotically like an inverse square. In the super-critical case, where there are infinitely many discrete eigenvalues, we compute precise asymptotics of the number of eigenvalues below a given energy $E$ as this energy tends to the bottom of the essential spectrum.
\end{abstract}

\section{INTRODUCTION}

This paper is concerned with discrete one-dimensional Schrödinger operators in $\ell^{2}\left(\mathbb{Z}_{+}\right)$, where $\mathbb{Z}_{+}=\{1,2,3, \ldots\}$. That is,

$$
H u(n)=-\Delta u(n)+V(n) u(n), \quad \Delta u(n)=u(n+1)-2 u(n)+u(n-1),
$$

where we impose a Dirichlet boundary condition, $u(0)=0$.

If $V(n) \rightarrow 0$ as $n \rightarrow \infty$, then zero is the bottom of the essential spectrum of $H$. We are interested in the discrete spectrum of $H$ below zero. Thus, for $E \geq 0$, we define

$$
N_{E}(H)=\operatorname{dim} \operatorname{Ran} P_{H}((-\infty,-E]),
$$

where $P_{H}$ is the family of spectral projections associated with $H$ by the spectral theorem. It is well known that $V(n) \sim-n^{-2+\varepsilon}$ produces finitely many eigenvalues if $\varepsilon<0$ and infinitely many if $\varepsilon>0$ and so inverse square decay is critical for the existence of infinitely many discrete eigenvalues below the essential spectrum. Furthermore, by the discrete analogue of Kneser's theorem, the discrete spectrum below zero of the operator $H$ with potential $V(n)=-c n^{-2}$ is finite when $c \leq \frac{1}{4}$ and infinite when $c>\frac{1}{4}$. Interestingly this discrete analog is much harder to prove and is not so well known. This was first shown by Năman [9] and later rediscovered in [2] (see also 8] for a generalization).

Our goal is to study the behavior of $N_{E}(H)$ as $E \downarrow 0$ in the super-critical case $c>\frac{1}{4}$. This question is natural since this case describes the borderline behavior at the transition between finitely and infinitely many bound states as described in the previous paragraph. In addition, we were also motivated by recent results on a connection between singular spectrum embedded in the essential spectrum

Received by the editors September 3, 2005 and, in revised form, November 9, 2005.

2000 Mathematics Subject Classification. Primary 47B36, 81Q10; Secondary 39A11, 47B39.

Key words and phrases. Discrete Schrödinger operators, bound states, oscillation theory.

This work was supported by the National Science Foundation under Grant No. DMS-0500910 and the Austrian Science Fund (FWF) under Grant No. P17762.

(C)2006 American Mathematical Society 
and the discrete spectrum of a given Schrödinger operator. Let us sketch this connection briefly. For operators of the form $H=-\Delta+V$ on the whole line, that is, acting in $\ell^{2}(\mathbb{Z})$, it was shown by Killip and Simon that the absence of bound states implies that the potential vanishes identically [6]. In other words, $\sigma(H) \subseteq[0,4]$ implies $V \equiv 0$. Damanik et al. found a simple variational proof of this statement that also allowed them to extend the result to operators in $\ell^{2}\left(\mathbb{Z}^{2}\right)[1$. It does not hold in dimensions three or higher, nor on the half-line, as shown in [1]. The counterexamples in $\ell^{2}\left(\mathbb{Z}^{d}\right), d \geq 3$, are given by sparse non-decaying potentials, whereas a possible counterexample in $\ell^{2}\left(\mathbb{Z}_{+}\right)$is given by $V(n)=(-1)^{n} / n$. Damanik and Killip then showed that, on the half-line, the absence of bound states implies the absence of embedded singular spectrum. In fact, absence of embedded singular spectrum follows already when there are finitely many bound states [3]. This raised the natural question of what can be said when there are infinitely many bound states. Is it true that sufficiently fast decay, for example finiteness of the moments

$$
N_{\gamma}=\sum_{n \geq 1}\left(\operatorname{dist}\left(E_{n},[0,4]\right)\right)^{\gamma}
$$

implies the absence of embedded singular spectrum? Here, $\left\{E_{n}\right\}_{n \geq 1}$ denotes the discrete eigenvalues of $H$ outside the essential spectrum [0,4]. Note that the DamanikKillip result can be phrased as follows: $N_{0}<\infty$ implies that $\sigma_{\text {sing }}(H) \cap[0,4]=\emptyset$. Damanik et al. then found an example with 2 as an embedded eigenvalue (and hence $\left.\sigma_{\text {sing }}(H) \cap[0,4] \neq \emptyset\right)$ and $N_{\gamma}<\infty$ for every $\gamma>0$ [4] This example has the potential $V(n)=(1+\varepsilon)(-1)^{n} / n$ for some small $\varepsilon>0$. The core of the proof consists of using methods developed in [1] and 2] to compare this sign-indefinite potential with sign-definite ones, both of the form $-c_{j} n^{-2}$, for different constants $c_{1}, c_{2}>1 / 4$. This clearly motivates a study of the bound state structure of supercritical inverse square potentials. The estimates for their bound states derived in [4] were sufficient to prove the result mentioned above, but it is desirable to have a more complete understanding; in particular since in the continuous case, the bound state structure has been studied in detail earlier. Namely, Kirsch and Simon considered operators of the form $-\frac{d^{2}}{d x^{2}}+V$ and carried out an investigation of $N_{E}(H)$ for super-critical inverse square potentials [7] (see also Schmidt [10] for extensions). In the continuous case, a study of inverse square potentials is also motivated by the fact that they arise in the partial wave analysis of rotationally symmetric partial differential operators.

We will prove the discrete analogue of the Kirsch-Simon result in this paper. On the one hand, as explained above, this case is more relevant to the question raised in [4, Sect. 2], where the authors asked if the Kirsch-Simon result has a discrete analog. On the other hand, the proof of Kirsch and Simon uses some arguments that do not carry over directly to the discrete case: They scale the spatial variable and use exact solvability of the Euler differential equation. Spatial scaling is not possible in the discrete case and, while there exists a discrete Euler equation, it is not symmetric.

\footnotetext{
${ }^{1}$ It was later realized by Damanik and Remling that $N_{\gamma}<\infty$ does indeed put some restrictions on the embedded singular spectrum; see [5]. For example, if $N_{\gamma}<\infty$ for every $\gamma>0$, then the embedded singular spectrum is supported on a set of zero Hausdorff dimension.
} 
Theorem 1. Suppose

$$
V(n)=-\frac{c}{n^{2}}+W(n), \quad c>\frac{1}{4}
$$

where $W$ is a decaying sequence such that $N_{0}(-\Delta+\gamma W)<\infty$ for all $\gamma \in \mathbb{R}$. Then

$$
\lim _{E \downarrow 0} \frac{N_{E}(-\Delta+V)}{-\ln (E)}=\frac{1}{2 \pi} \sqrt{c-\frac{1}{4}} .
$$

Remarks. (i) We say that a sequence $W$ is decaying if $W(n) \rightarrow 0$ as $n \rightarrow \infty$.

(ii) The hypothesis on $W$ is satisfied, for example, if $\sum_{n>0} n|W(n)|<\infty$. See [12, Thm. 5.10].

(iii) An analogous result holds on the whole line. This follows quickly from the half-line result since the whole-line case can be reduced to the half-line case by Dirichlet decoupling.

(iv) For perturbations of the form $V(n)=\frac{c}{n^{2}}+W(n)$, an analogous result holds near the top of the essential spectrum.

\section{Proof of Theorem 1}

As preparation, we state the discrete analog of Proposition 5 from [7. The proof is analogous.

Lemma 2. Let $V, W$ be decaying sequences. Then for every $E>0$ and $0<\varepsilon<1$, we have

$$
\begin{aligned}
& N_{E}(-\Delta+V+W) \leq N_{E}\left(-\Delta+\frac{1}{1-\varepsilon} V\right)+N_{E}\left(-\Delta+\frac{1}{\varepsilon} W\right), \\
& N_{E}(-\Delta+V+W) \geq N_{E}(-\Delta+(1-\varepsilon) V)-N_{E}\left(-\Delta-\frac{1-\varepsilon}{\varepsilon} W\right) .
\end{aligned}
$$

Now we come to the proof of our main theorem. We start with

$$
V_{c}(n)=-\frac{c}{n^{2}}
$$

and replace it by $V_{E, c}$, which is just $V_{c}-E$ on $\left\{n: V_{c}(n) \leq-E\right\}$ and equal to $V$ otherwise. To investigate the asymptotics of $N_{E}\left(-\Delta+V_{E, c}\right)$, we split our domain into two parts by cutting at $\sqrt{\frac{c}{E}}$. For the first part, we will compute the asymptotics of $N_{E}$ directly. The remaining part does not contribute to $N_{E}$. Then we use Lemma 2 to show that $N_{E}$ has the same asymptotics for $V_{E, c}$ and $V=V_{c}+W$.

Lemma 3. We have

$$
\lim _{E \downarrow 0} \frac{N_{E}\left(-\Delta+V_{E, c}\right)}{-\ln (E)}=\frac{1}{2 \pi} \sqrt{c-\frac{1}{4}} .
$$

Proof. We first decompose $-\Delta+V_{E, c}$ into two parts by imposing an additional Dirichlet boundary condition at $\left\lfloor\sqrt{\frac{c}{E}}\right\rfloor$. Since this constitutes a rank-one resolvent perturbation, it will not affect the limit. By the choice of our cut point, the part with $n>\left\lfloor\sqrt{\frac{c}{E}}\right\rfloor$ does not contribute and by oscillation theory (see, e.g., 11] or [12, Ch. 4]) it suffices to count the number of sign flips of some solution of $\left(-\Delta+V_{E, c}\right) u=$ $-E u$ on $\left(1, \sqrt{\frac{c}{E}}\right)$, that is, the number of sign flips of some solution of $\left(-\Delta+V_{c}\right) u=0$ on $\left(1, \sqrt{\frac{c}{E}}\right)$.

Unfortunately, $\left(-\Delta+V_{c}\right) u=0$ is not explicitly solvable, but

$$
\tilde{u}_{c}(n)=\sqrt{n} \exp \left(\mathrm{i} \sqrt{c-\frac{1}{4}} \ln (n)\right)
$$


solves $\left(-\Delta+\tilde{V}_{c}\right) \tilde{u}=0$ with the complex-valued potential

$$
\tilde{V}_{c}(n)=\frac{\Delta \tilde{u}_{c}(n)}{\tilde{u}_{c}(n)}=-\frac{c}{n^{2}}+O\left(\frac{1}{n^{3}}\right) .
$$

Moreover, it is straightforward to check (cf. [12, Lemma 7.10], resp. 8]) that $-\Delta u+V_{c} u=0$ has a solution $u_{c}$ which asymptotically looks like $\tilde{u}_{c}(n)$. Taking the real part of $u_{c}$, we see that the number of sign flips behaves to leading order like $-\frac{1}{2 \pi} \sqrt{c-\frac{1}{4}} \ln (E)$.

Let us prove the upper bound in (1.1). By Lemma 2,

$$
\begin{aligned}
N_{E}\left(-\Delta+V_{c}+W\right) & =N_{E}\left(-\Delta+\left(V_{c}-\chi_{(1-\varepsilon) E, c}\right)+N_{E}\left(\chi_{(1-\varepsilon) E, c}+W\right)\right) \\
& \leq N_{E}\left(-\Delta+V_{E, c /(1-\varepsilon)}\right)+N_{E}\left(-\Delta+\frac{1}{\varepsilon}\left(\chi_{(1-\varepsilon) E, c}+W\right)\right),
\end{aligned}
$$

where $\chi_{E, c}=E \chi_{(0, \sqrt{c / E})}$ and $\chi_{\Omega}$ is the characteristic function of the set $\Omega$. Using

$$
N_{E}\left(-\Delta+\frac{1}{\varepsilon}\left(\chi_{(1-\varepsilon) E, c}+W\right)\right) \leq N_{0}\left(-\Delta+\frac{1}{\varepsilon} W\right),
$$

the assumption on $W$, and Lemma 3, we see that

$$
\limsup _{E \downarrow 0} \frac{N_{E}\left(-\Delta+V_{c}+W\right)}{-\ln (E)} \leq \frac{1}{2 \pi} \sqrt{\frac{c}{1-\varepsilon}-\frac{1}{4}}
$$

for every $0<\varepsilon<1$, that is,

$$
\limsup _{E \downarrow 0} \frac{N_{E}\left(-\Delta+V_{c}+W\right)}{-\ln (E)} \leq \frac{1}{2 \pi} \sqrt{c-\frac{1}{4}}
$$

It remains to show the lower bound in (1.1). By Lemma 2 ,

$$
\begin{aligned}
N_{E}\left(-\Delta+V_{c}+W\right) & =N_{E}\left(-\Delta+\left(V_{c}-\chi_{E /(1-\varepsilon), c}\right)+N_{E}\left(\chi_{E /(1-\varepsilon), c}+W\right)\right) \\
& \geq N_{E}\left(-\Delta+V_{E,(1-\varepsilon) c}\right)-N_{E}\left(-\Delta-\frac{1-\varepsilon}{\varepsilon}\left(\chi_{E /(1-\varepsilon), c}+W\right)\right) .
\end{aligned}
$$

Observe that it suffices to show that the second summand does not contribute to the limit. Invoking Lemma 2 a second time, we have

$$
N_{E}\left(-\Delta-\frac{1-\varepsilon}{\varepsilon}\left(\chi_{E /(1-\varepsilon), c}+W\right)\right) \leq N_{E}\left(-\Delta-\frac{1}{\varepsilon} \chi_{E /(1-\varepsilon), c}\right)+N_{E}\left(-\Delta-\frac{1-\varepsilon}{\varepsilon^{2}} W\right) .
$$

The second term is bounded for fixed $\varepsilon$ as $E \downarrow 0$ by assumption and it remains to investigate the first one. As before, we impose a Dirichlet boundary condition at $\left\lfloor\sqrt{\frac{c(1-\varepsilon)}{E}}\right\rfloor$ and we need to count the sign flips of the solution of $-\Delta u-\frac{E}{\varepsilon(1-\varepsilon)} u=$ $-E u$ on $\left(0, \sqrt{\frac{c(1-\varepsilon)}{E}}\right)$. Since this equation is explicitly solvable we obtain

$$
N_{E}\left(-\Delta-\frac{1}{\varepsilon} \chi_{E /(1-\varepsilon), c}\right)=\sqrt{c\left(1-\varepsilon-\frac{1}{\varepsilon}\right)}+O(E) .
$$

Hence

$$
\liminf _{E \downarrow 0} \frac{N_{E}\left(-\Delta+V_{c}+W\right)}{-\ln (E)} \geq \frac{1}{2 \pi} \sqrt{(1-\varepsilon) c-\frac{1}{4}}
$$

for every $0<\varepsilon<1$, and thus,

$$
\liminf _{E \downarrow 0} \frac{N_{E}\left(-\Delta+V_{c}+W\right)}{-\ln (E)} \geq \frac{1}{2 \pi} \sqrt{c-\frac{1}{4}} .
$$

Combining (2.1) and (2.2), we obtain the assertion of the theorem. 


\section{ACKNOWLEDGMENTS}

Gerald Teschl gratefully acknowledges the extraordinary hospitality of the Department of Mathematics at Caltech, where this work was done.

\section{REFERENCES}

[1] D. Damanik, D. Hundertmark, R. Killip, and B. Simon, Variational estimates for discrete Schrödinger operators with potentials of indefinite sign, Commun. Math. Phys. 238, 545-562 (2003). MR1993385 (2004i:81068)

[2] D. Damanik, D. Hundertmark, and B. Simon, Bound states and the Szego" condition for Jacobi matrices and Schrödinger operators, J. Funct. Anal. 205, 357-379 (2003). MR 2017691 (2005i:81180)

[3] D. Damanik and R. Killip, Half-line Schrödinger operators with no bound states, Acta Math. 193, 31-72 (2004). MR2155031

[4] D. Damanik, R. Killip, and B. Simon, Schrödinger operators with few bound states, Comm. Math. Phys. 258, 741-750 (2005). MR2172016 (2006e:47069)

[5] D. Damanik and C. Remling, Schrödinger operators with many bound states, to appear in Duke Math. J.

[6] R. Killip and B. Simon, Sum rules for Jacobi matrices and their applications to spectral theory, Ann. of Math. 158, 253-321 (2003). MR.1999923 (2004f:47040)

[7] W. Kirsch and B. Simon, Corrections to the classical behavior of the number of bound states of Schrödinger operators, Ann. Phys. 183, 122-130 (1988). MR0952875 (90b:35065)

[8] F. Luef and G. Teschl, On the finiteness of the number of eigenvalues of Jacobi operators below the essential spectrum, J. Difference Equ. Appl. 10, 299-307 (2004). MR2049680 (2005b:39017)

[9] P. B. NaĬman, The set of isolated points of increase of the spectral function pertaining to a limit-constant Jacobi matrix (Russian), Izv. Vysš. Učebn. Zaved. Matematika 8, 129-135 (1959). MR0131776(24:A1624)

[10] K. M. Schmidt, Critical coupling constants and eigenvalue asymptotics of perturbed periodic Sturm-Liouville operators, Comm. Math. Phys. 211, 465-485 (2000). MR1754525 (2001i:34147)

[11] G. Teschl, Oscillation theory and renormalized oscillation theory for Jacobi operators, J. Diff. Eqs. 129, 532-558 (1996). MR1404392 (98m:47053)

[12] G. Teschl, Jacobi Operators and Completely Integrable Nonlinear Lattices, Math. Surv. and Mon. 72, Amer. Math. Soc., Rhode Island, 2000. MR1711536 (2001b:39019)

Mathematics 253-37, California Institute of Technology, Pasadena, California 91125 E-mail address: damanik@caltech.edu

URL: http://www.math.caltech.edu/people/damanik.html

Faculty of Mathematics, Nordbergstrasse 15, 1090 Wien, Austria - And - International Erwin Schrödinger Institute for Mathematical Physics, Boltzmanngasse 9, 1090 Wien, Austria

E-mail address: Gerald.Teschl@univie.ac.at

URL: http://www.mat.univie.ac.at/ gerald 